\title{
Estimativa de Modelos de Preços Hedônicos para Locação Residencial em Porto Alegre
}

\section{Marco Aurélio Stumpf González}

Eng. Civil, M.Sc.; Professor Substituto UFRGS

\section{Carlos Torres Formoso}

Eng. Civil, Ph.D.; Professor Adjunto UFRGS

Universidade Federal do Rio Grande do Sul

Curso de Pós-Graduação em Engenharia Civil - CPGEC/NORIE

Av. Osvaldo Aranha, 99/3 $3^{\circ}$ Andar - 90210-000 - Porto Alegre - RS

Palavras-Chave: mercado imobiliário; locação de imóveis; modelọs de preços hedônicos.

Key Words: housing market; rental housing; hedonic price models.

\section{RESUMO}

Este artigo descreve una pesquisa cujo objetivo è a busca de modelos estatísticos de múltiplas variáveis, que expliquem a formação de valor de aluguel de apartamentos residenciais em Porto Alegre. A amostra ś composta por apartamentos ofertados em junho de 1992, tendo cono universo todos os imóveis oferecidos no periodo. Do total de 1819 unidades, foram selecionados 504 imóveis, nos quais foram investigadas diversas características, tais como estado do prédio a condições do entomo, complementadas por informações de órgãos públicos. Os dados obtidos foram testados através de análise fatorial e de regressão, com a compilação de modelos econométricos (hedônicos), em vários niveis de agregação. A análise demonstrou que o mercado pode ser explicado através de modelos deste tipo, que apresentam diferenças significativas, confome o subconjunto de dados, mas permitem a obtenção de modelos para todos os imóveis e um melhor entendimento do funcionamento do mercado imobiliário.

\section{ABSTRACT}

This research work aim at investigating the behavior of the real estate market of the city of Porto Alegre. through the use of multiple variable statistical models, which explain the value formation of housing rents. The models cleveloped were based on a sample of 504 apartments, from several different areas of the city, offered in the market during July 1992. The universe is all properties offered in that period. The models developed were based on a sample of 50 t apartments, extracted of the total of 1819 . In that apartments, a number of variables had their influence in the value of rent investigated, like building condition and proximity conditions. completed by information of public institutions. The data were tested by factor and regression analysis, with the compilation of econometric models (hedonic), in varions aggregation levels. The analysis demonstrated what the market can to be explicated by this models, what present significalive differences, according to data collection, but permit the obtainment of models for all dwellings and a best understanding of working of real estate market. 


\section{Introdução}

Existe a necessidade de pesquisar a natureza das variáveis mais importantes na formação do valor de aluguéis, cmpregando-se o processo estatístico adequado para analisar o fenômeno. É importante conhecer os mecanismos de formação de valor, com o propósito de obter informações para as entidades ligadas à questão da habitação, além das finalidades diretas, como avaliação do valor de mercado. O desenvolvimento de tais estudos pode auxiliar a definição das medidas de planejamento urbano c projetar a influĉncia destas no mercado.

A hipótese principal do estudo é a cxistência de um modelo único para a cidade, ou seja. um modelo que permita cxplicar a formação de valor dos alıguéis para qualquer imóvel do mercado. Por outro lado, sc não for possível encontrar este modelo único, cxiste a possibilidade de desenvolver-se modelos diferenciados para scgmentos do mercado, por regiões ou tipo de imóvel. Uma hipótese alternativa é a de que existem algumas variáveis que participam de qualquer modelo c diversas outras que têm importância conforme o tipo de imóvel.

O objctivo geral do trabalho ć contribuir para o conhecimento sobre o mercado imobiliánio. especialmente com relação à locação de apartamentos residenciais $\mathrm{cm}$ Porto Alegre, c sobre os processos técnicos adolados na análise da formação dos valores. Neste artigo aprescnta-se uma sintese do estudo desenvolvido. Maior detalhamento sobre os procedimentos de amostragem e análise estatística, com a apresentação de outros modelos, poderá ser encontrado cm González (1993).

\section{Mercado Imobiliário}

O mercado de habitações é dominado pelo estoque, por causa da durabilidade das construções. Este estoque reflete decisões tomadas no passado e a heterogeneidade das construçõos indica a variação, ao longo do tempo, dessas decisões. Existe uma relação direta entre preços de habilações novas c existentes. A oferta consiste principalmente de imóveis do estoque, sendo que as novas construções representam um percentual relativamente pequeno do total, c por isso a oferta de habitações tende a ser inelástica. Desta forma, as mudanças de preço são causadas predominantemente por mudanças de demanda (BALCHIN, 1981; BALCHIN \& KIEVE, 1986; LAVENDER, 1990; LEAN \& GOODALL, 1966).

Dada a imobilidade das construções, o cxcedente de oferta em uma determinada região não pode ser deslocaldo para compensar a falta em outra. Por isso, o equilibrio de demanda entre diferentes bairros ou cidades se dá, à curto prazo, unicamente via preços. Além disso, o mercado tende a ser limitado $\mathrm{cm}$ cxtensão, dentro de uma cidade ou atć em parte dela: é geograficamente bascado. Existem variações regionais de demanda, oferta $\mathrm{c}$ preço (LAVENDER, 1990); WEIMER \& HOYT, 1948). 
Há uma considerável variedade de produtos: os imóveis apresentam uma clcvada heterogeneidade, atć para um mesmo prédio ou localização. Exisıem também grandes variações de preço entre imóveis semelhantes, dependendo de sua localização. A explicação mais comum para isso é a variação no valor dos terrenos onde as habitações são construídas. Entretanto, na prática cxiste uma sćric de fatores, além dos terrenos, que contribuem para diferenciar os preços dos imóveis (LAVENDER, 1990, ROBINSON, 1979).

Porém, a localização tem influência significativa nos preços das liabitações. Em geral considera-se a distância ao centro comercial/histórico da cidade. Porém, sc for considerado o caso geral, em que o acesso a diversos pontos da cidade (universidades, hospitais, grandes centros de compras, locais de lazer) pode ser de interesse para um grande numero de pessoas, a análise do fenômeno sc complica c as alterações de preço dos imóvcis não terĩo uma relaçĩo linear com as alıcrações de distância (BALCHIN \& KIEVE, 1986: LEAN \& GOODALL, 1966).

Foi verificado cmpiricamente que o preço do imóvel pode ser relacionado com o fluxo de serviços de habitação que este imóvel gera ou, cm outras palavras, com suas caracteristicas. É possivel dividir-se a habilação em um número limilado de caracteristicas ou serviços dos quais cla é composta, e cstes assumem valores específicos no mercado de acordo com a demanda (utilidade gerada no consumo de cada serviço scparadamente) c a oferta (disponibilidade) dos mesmos. Os indivíduos valorizam os diversos atributos da habilação de forma diferenciada e, de modo geral, os preços implicitos de cada característica ou scrviço da habitação tendem a variar diretamente com a oferta dos mesmos (LUCENA, 1985).

\section{Métodos de Avaliação e Amostragem}

Produtos diferenciados, como os imóveis, podem ser descritos por vetores de características medidas objetivamente. Os preços dos produtos observados c as quantidades especificas das caraclerísticas associadas com cada bem definem um conjunto de preços implícitos ou hedônicos. A teoria dos preços hedônicos pode ser entendida como um problema de equilibrio cspacial econômico, com múltiplas variáveis influentes (ROSEN, 1974; WITTE et all, 1974).

As annilises microeconômicas cmpíricas procuram modelos econométricos da formação de preços, conhecidos por "hedonic housing price equations", os quais buscam explicar diferenças nos preços através de variações nas caractcrísticas das habilações (LUCENA, 1985).

Os modelos deste estudo são baseados em equaçôes de regressĩo de múltiplas variáveis. Os cocficientes das cquações podem ser interpretados como preços implícilos de uma unidade de cada caracteristica considerada no modelo cconométrico. Esses modelos sĩo utilizaldos na avaliação de imóvcis para encontrar o valor de mercado $\mathrm{cm}$ um 
determinado momento, de acordo com os dados da amostra: A finalidade básica ć a medição dos scrviços de habitação, através de variáveis como área, localização, idade do prédio, vizinhança, c outros. Os modelos podem fornecer uma cstimativa de valor de um imóvel, através da substituição das caracterislicas particulares do imóvel no modelo desenvolvido (FRANCHI, 1991; LUCENA, 1985).

Em geral, os csludos sobre o mercado imobiliário $t \mathrm{endem}$ a reunir variáveis colineares, por causa da dificuldade de mediçĩo c da inter-relação cntre as caractcrísticas consideradas (por cxemplo: sociais, cconômicas, fisicas e geogríficas). Para reduzir os efeitos da colinearidade, foi cmpregada a técnica de análise fatorial, que agnupa em algumas variáveis independentes (fatores) as influências aprescntadas pelo conjunto de variáveis $\mathrm{em}$ análise. A participação simultânea $\mathrm{cm}$ um fator permite identificar variáveis colineares. Desta forma, apenas uma variável de cada Fator cra incluida na equação (HARMANN, 1976: MORTON, 1977; WILKINSON \& ARCHER, 1973).

\section{Determinação da Amostra de Trabalho}

Quando os dados são muito hetcrogêncos (como é o caso do mercado imobiliário), o processo pelo qual se obtém a amostra torna-se crítico $c$ o csludo das técnicas que assegurem amostras dignas de confiançá ć importantc. Da mesma forma, uni dos pontos fundamentais da inferĉncia estatistica é precisamente o da escolla da amosira (COCHRAM, 1965; KONIJN, 1973: RAJ, 1972; SUDMAN, 1976).

Em função da inevistência de dados organizados que servissem como base para o projeto da amostra, foi definida um pesquisa buscando a enumeração completa da oferta $\mathrm{em}$ um período determinado. Os dados para análise compõe-se de imóvcis ofertados ao mercado no mês de junho de 1992 , tomando-se como valor de aluguel o que foi ofertado. A intenção da pesquisa foi abranger toda a oferla de apartamentos residenciais na cidade de Porto Alegre, neste periodo. Naturalmente, os imóveis do mercado informal estão cxcluídos. Os imóveis foram identificados cm consultas dirclas às imobiliárias $\mathrm{c} \mathrm{cm}$ anúncios dc jornais. Verificou-se que apenas uma fraçĩo dos imóveis oferlados ao mercado pelas imobiliárias não são anunciados nos jormis.

Como se pretende analisar a cidade intcira, a amostra deve contcmplar todas as regiōes. Há consenso na litcratura sobre cconomia urbana accrca da importância da localização para a fonlação do preço dos imóvcis (por cxcmplo: BALCHIN, 1981; FRANCHI,1991; KAIN \& QUIGLEY, 1970; LEAN \& GOODALL, 1972; MUTH, 1975; ROBINSON, 1979; WEIMER \& HOYT, 1948). Assim, o bairro onde os imóvcis se localizam foi cscolhido como parâmetro para a organização $\mathrm{cm}$ grupos. Os imóveis foram agnupados scgundo os limites oficiais (legislação municipal) dos bairros a que pertencem.

A partir desics dados foi realizada a 
scleção da amostra, pelo mélodo de agrupamento de imóvcis pelos bairros. Alguns bairros apresentaram número insuficiente de imóveis para análise c foram unidos a bairros vizinhos, procurando-sc bairros semelhantes, em termos de renda média familiar, de acordo com o Censo de 1980 (FIBGE), status c condições gerais. Foram incluidos nesla situação os bairros com menos de dez unidades. Em seguida, foram cxtraídas amostras de cada gnıpo, individualmente, com fração amostral constante de (aproximadamente) 500/1815. Estes imóveis constituem a amostra final, analisada detidamente: para os clcmentos desta amostra, foram realizadas vistorias cxternas aos prédios, verificando-se padrão de acabamento, idade aparente $\mathrm{c}$ material da fachada. Tambćm realizou-sc um lcvantamento das características do cntomo: urbanização (accsso, pavimentação) $c$ scrviços públicos (água, luz telcfonc). De uma forma geral, todos os imóveis se equivalem quanto a estes dois últimos aspectos. Alćm disso, foi cfectuada uma consulta à Prefeitura Municipal, junto ao cadastro da Secretaria Municipal da Fazenda (SMF), onde foram obtidas a área total $\mathrm{c}$ a idade do imóvel.

\section{Análise Estatística}

Concluida a fase de obtenção dos dados, foi definido um conjunto de variáveis, buscando-se representar as caracteristicas obscrvadas dos imóvcis de forma cxtensiva. Sobre csic conjunto foi realizada análise fatorial, com o intuito de reunir variávcis com comportamento scmellamte, reduzindo os riscos de multicolinearidade $\mathrm{c}$ facilitando a análise (HARMANN, 1976; MORTON, 1977; WILKINSON \& ARCHER, 1973).

Para a análise dos dados foram claboradas regressões cruzando as variávcis dependentes com as variáveis independentes. Foram testados modelos incluindo uma das variáveis dependentes (alugućis cm CRUZEIRO ou DOLAR), uma das independentes que representa $o$ tamanho (ÁREA do imóvel ou número de QUARTOS), c todas as outras variávcis. testando cste conjunto pelo método "stepivise" (NIE et all, 1975; WEISBERG, 1985).

Os resultados das análises cfetuadas cstão aprescntados a seguir. Para os modelos ajustados, as tabelas apresentam as variáveis sclecionadas, seus coeficientes c a indicação da significância. Além disso. são incluidos indicadores do ajustamento, como coeficiente de determinação $\left(\mathbf{R}^{2}\right)$, teste de variância $(\boldsymbol{F})$, número de regressores (k), número de imóvcis do conjunto testado (n) c número de "outliers" (residuos acima de dois desvios-padrĩo, em módulo). Os valores de $\mathbf{F}$ para os modelos compilados neste trabalho ficaram sempre muito acima nos limites mínimos indicados pela Norma Brasileira de Avaliação de Imóvcis Urbanos (ABNT, 1989), que foram cmpregados como referência.

Para verificar as fugas das condições básicas admitidas para a análisc realizada, form constnuídos gráficos investigativos dos resíduos, comparando-os com a curva Normal c com os valores estimados pelas equações. A análise dos gráficos não indicou 
qualquer problema grave nos modelos (JOHNSTON, 1977; KMENTA, 1978; MADDALA, 1977; NETER \& WASSERMANN, 1974; NIE et all, 1975).

\section{Regressões com o Conjunto Total de Dados}

Inicialmente foram claboradas regressões envolvendo o valor total $\mathrm{c}$ as variáveis ÁREA c QUARTOS. Os modelos ajustados, aprescntades na Tabela 1 , mostraram-se adequados, com bons cocficientes de determinação (cerca de 80\%). Tanto nas cquações com ÁREA como naquelas com QUARTOS, os resultados são semelhantes. As variáveis cnvolvidas $\mathrm{cm}$ geral foram as mesmas, com valores $\mathrm{c}$ sinais dos coeficientes normais, dentro do comportamento csperado.

As regressões cm CRUZEIRO (Cr\$1 c Cr\$2) aprescntam a variável DIA (dala da ofcra), chquanto aquelas cm que a variável dependente ć o valor tolal em dólares americanos (US\$1 e US\$2) não incluem esta variável, indicando que a influência do tempo seria decorrente da correção monetária dos valores, c não de uma valorização real no pcríodo.

De uma forma geral, esperava-se a participação da distância ao centro da cidade, conforme indicação da bibliografia consultada. Nos modelos com QUARTOS, aparecem as variávcis DCENTRO c DSHOP, que represcntam as distâncias ao centro $\mathrm{c}$ aos dois maiores "shopping cenlers" da cidade.

\begin{tabular}{|c|c|c|c|c|c|}
\hline VARIÁVELL. & FATOR & CIs $(1)$ & C.IS(2) & USSS(1) & USS(2) \\
\hline ÁREA & 1 & $4+12$ & & 1.268 & \\
\hline QUARTOS & 1 & & 130166,09 & & 37.333 \\
\hline BANHEIROS & 2 & 79241 & & 23.226 & $17.692^{\circ}$ \\
\hline APTOS & 3 & $-211^{*}$ & & & \\
\hline NAA & 3 & & & $-1 .(k) 1 *$ & \\
\hline USOD & + & $-31626^{*}$ & & $-7.491 *$ & \\
\hline DIA & 6 & 2401 & 2719 & & \\
\hline ESTADO & 7 & & $1 \$ 36$ & & 0.430 \\
\hline IARDIM & $x$ & 21028 & & 7.061 & $4.009^{\circ}$ \\
\hline DCENTRO & 9 & & & & -2.908 \\
\hline DSIIOP & 9 & & -13885 & & \\
\hline DSUPER & 9 & 11124 & & 3.077 & \\
\hline BAIRRO & - & 1568 & 2211 & 0.437 & 0.533 \\
\hline $\mathrm{BOX}$ & - & 73161 & 136927 & 22.500 & 40.897 \\
\hline COBERIURA & $\cdot$ & 1100465 & $16 x, 22+0$ & 297.839 & $462.37 y$ \\
\hline DEPEMP & - & 60271 & 88433 & 19.161 & 24.782 \\
\hline FAVD & - & -118922 & .127067 & .19 .604 & -41.862 \\
\hline MOBILIA & - & 75429 & 105009 & 22.997 & 30.565 \\
\hline TIPO & - & +2005 & 77420 & 11.225 & 21.279 \\
\hline CONSTANTE & & -246666 & -204764 & -49.786 & -57.735 \\
\hline$R^{2}$ & & 0.827 & 0.788 & 0.824 & 0.789 \\
\hline $\mathbf{F}$ & & 167.021 & 166.504 & 177.573 & 153.465 \\
\hline $\mathbf{n}$ & & 504 & 504 & 504 & 504 \\
\hline$k$ & & 14 & 11 & 13 & 12 \\
\hline "OUTI.IERS" & & 11 & 15 & 12 & 14 \\
\hline
\end{tabular}

(nível de significância: *:5\%; ontros:1\%)

Tabela 1 - Regressões Empregando o Conjunto Total de Imóveis 
Nos modelos nos quais sc utilizou a ÁREA, a variável que foi incluida nas cquaçõcs foi DSUPER, com sinal contrírio ao esperado. Neste caso, a variável pode estar indicando situações inconvenientes relacionadas com a proximidade de locais mais movimentados, tais como nuido $\mathrm{c}$ congestionamentos de trânsito.

\section{Regressões com Sub-Grupos}

Para testar a hipótese da cxistência de um modelo único, foram montadas cquações bascadas cm parcclas do mercado, comparando-as com os modelos que cmpregaram o conjunto total da amostra. Como scrá visto a seguir, estes modelos são diferenciados daqueles, $\mathrm{cm}$ quantidade $\mathrm{c}$ tipo de variáveis. Algumas difcrenças devem-se a restrições dos dados, como a não cxistência de um tipo de imóvel na parcela considerada, o que acarreta a cxclusão de variáveis. São apresentados a seguir as equações com os imóveis divididos por número de dormilórios $\mathrm{c}$ por distância ao centro.

Agrupamento por Número de Dormitórios

O conjunto total de imóveis foi dividido $\mathrm{cm}$ dois gnupos, considcrando-sc o número de dormilórios, buscando separar os imóveis pequenos dos grandes. Assim, foram feitas regressões com imóveis tipo JK e de um domitório c, de outro lado, imóveis de dois, três e quatro dormitórios. A diferenciação de modelos estatísticos cntre os dois grupos ć cvidente,

\begin{tabular}{|l|c|r|r|r|r|}
\hline VARIÁVEL & FATOR & \multicolumn{1}{c|}{ Cr $\$(1)$} & \multicolumn{1}{c|}{ Cr\$(2) } & \multicolumn{1}{c|}{ US\$(1) } & \multicolumn{1}{c|}{ US\$(2) } \\
\hline ÁREA & 1 & & 3042 & & 0.892 \\
\hline QUARTOS & 1 & 99614 & & 29.212 & \\
\hline APTOS & 3 & & -163 & & -0.047 \\
\hline ESQUINA & 5 & $-22979^{*}$ & & $-6.612^{*}$ & \\
\hline DIA & 6 & 1318 & 1810 & $-0.281^{*}$ & \\
\hline QUALIDADE & 7 & 812 & & 0.240 & \\
\hline JARDIM & 8 & & $10614^{*}$ & & 3.175 \\
\hline DCENTRO & 9 & & -6049 & & -1.915 \\
\hline DSHOP & 9 & -15529 & & -4.521 & \\
\hline BOX & - & 45616 & $34927^{*}$ & 14.142 & 11.527 \\
\hline MOBILIA & - & 70025 & 73046 & 21.219 & 22.387 \\
\hline CONSTANTE & & 198602 & 123467 & 71.833 & 47.328 \\
\hline R $^{2}$ & & 0.561 & 0.510 & 0.546 & 0.494 \\
\hline F & & 48.090 & 39.154 & 45.258 & 42.957 \\
\hline$n$ & & 271 & 271 & 271 & 271 \\
\hline $\mathrm{k}$ & & 7 & 7 & 7 & 8 \\
\hline "OUTLIERS" & & 10 & 16 & 9 & 15 \\
\hline
\end{tabular}

(nível de significancia: *:5\%; demais: 1\%)

Tabela 2 - Regressões em CRS e USS para Imóveis Pequenos (Jk e um Dormitório) 
principalmente porque se tratam de dois segmentos de mercado distintos c porque os imóvcis menores têm menor variação de características, ou scja, menos opções.

Foram montadas regressões de dois tipos: com QUARTOS c ÁREA. Inicialmente, para o grupo dos imóvcis $\mathrm{JK} / \mathrm{ID}$, os cocficientes de detcrminação foram baixos (cerca de $50 \%$ ), cmbora as variáveis cuvolvidas scjam, na maioria, as mesmas que na análise anterior. Os resultados desta análise estão na Tabela 2.

Eim scguida, foram selecionados os imóvcis com 2/3/4D. As cquações montadas com o conjunto de dados testado apresentam pouca semelhança de coeficientes $\mathrm{c}$ variáveis envolvidas, $\mathrm{cm}$ relação aos modelos de JK/ID, demonstrando as diferenças enitre estes dois tipos básicos de imóvel. Os cocficientes de detcrminação, aprescntados na Tabcla 3 , foram melhores, assim como os testes $\mathbf{F}$ c o número de "outliers".

O ajustamento dos modelos aprescntados foi satisfatório, com bons coeficientes de determinação e poucos "oullicrs". A comparação das equações com os imóveis divididos por número de dormitórios permitiu identificar dois modelos distintos. Provavelmente isto se deve às difcrenças de opções de tipos de imóvcl. ou scja, imóvcis pequenos não têm duas salas, dependências de empregada ou cobcrtura, limitando a escolha dos usuários

\begin{tabular}{|c|c|c|c|c|c|}
\hline VARIÁVEL & FATOR & $\operatorname{Cr} S(1)$ & $\operatorname{CrS}(2)$ & USS (1) & US\$(2) \\
\hline ÁREA & 1 & & 3959 & & 1.066 \\
\hline QUARTOS & 1 & 168037 & & 51.412 & \\
\hline USOD & 4 & $-45939 *$ & & & \\
\hline DIA & 6 & 3466 & 3351 & & \\
\hline ESTADO & 7 & 2146 & & 0.670 & $0.352^{*}$ \\
\hline JARDIM & 8 & & $20557^{*}$ & & \\
\hline PRÉDIOS & 8 & & & $-1.652^{*}$ & \\
\hline DSUPER & 9 & & 15268 & & 4.325 \\
\hline BAIRRO & - & 3721 & 2522 & 0.832 & 0.639 \\
\hline $\mathrm{BOX}$ & - & 165294 & 115629 & 48.005 & 36.530 \\
\hline COBERTURA & - & 1411775 & 1004749 & 401.809 & 289.636 \\
\hline FAVD & - & $-113099^{*}$ & $-116970^{*}$ & $-35.509^{*}$ & $-28.700^{* *}$ \\
\hline MOBILIA & - & 193861 & & 57.750 & $28.156^{124}$ \\
\hline TIPO & - & 125516 & 85038 & 33.641 & 23.584 \\
\hline CONSTANTE & & .538869 & -325017 & -128.760 & -70.839 \\
\hline $\mathrm{R}^{2}$ & & 0.787 & 0.813 & 0.780 & 0.808 \\
\hline $\mathrm{F}$ & & 82.216 & 108.169 & 88.155 & 104.282 \\
\hline 11 & & 233 & 233 & 233 & 233 \\
\hline $\mathrm{k}$ & & 10 & 9 & 9 & 9 \\
\hline "OUTLIIERS" & & 9 & 8 & 9 & 7 \\
\hline
\end{tabular}

(nivel de significância: **: $10 \% ; *: 5 \%$; demais: $1 \%$ )

Tabela 3 - Regressões em CRS e USS para Imóveis Grandes (dois, três e quatro dormitórios) 
c as variações de prę̧o cntre os imóvcis, os quais são mais semelhantes que os imóvcis maiores. Desta forma, as variácis respectivas são constantes para cstes imóveis c não participam dos modelos, ao contrário dos modclos para imóveis maiores.

\section{Agrupamento por Distância do Centro}

Nesla forma, foi identificada a mediana das distâncias, dividindo-se o conjunto total cII dois gnupos de mesmo tamanho (252 unidades). Para o gnupo mais próximo do centro (distâncias de alć $2,24 \mathrm{~km}$ ), não cxistem na amostra imóveis com cobertura, ou scja, a variável COBERTURA assume valor constantc 0 . Os modelos aprescntam algumas difcrenças, cm relação aos modclos iniciais, com variáveis c cocficientes diferentes, conforme indican as Tabelas 4 c 5 , a seguir.

Para os imóveis mais distantes do centro da cidade, a variável IDADE apresentou sinal positivo nas cquações com ÁREA, ao contrário do gripo de imóvcis mais próximos ao centro, o que provavelmente deve-se a $\mathrm{um}$ efeito de coline raridade entre as variávcis, específico para o grupo de imóveis considerado. Como cste cfeito não pode ser aceito, invalida os modelos a variável foi climinada das equaçõcs em que surgiu com este comportamento.

\begin{tabular}{|l|r|r|r|r|r|}
\hline VARIÁVEL & FATOR & \multicolumn{1}{|c|}{ Cr\$(1) } & \multicolumn{1}{c|}{ Cr\$(2) } & \multicolumn{1}{c|}{ US\$(1) } & \multicolumn{1}{c|}{ US\$(2) } \\
\hline AREA & 1 & & 3956 & & 1.125 \\
\hline QUARTOS & 1 & 134889 & & 39.154 & \\
\hline NAA & 3 & & $-3518^{*}$ & & $-1.110^{*}$ \\
\hline PAVIMENTO & 4 & & $34443^{*}$ & & $9.156^{*}$ \\
\hline DIA & 6 & 2498 & 2382 & & \\
\hline IDADE & 7 & $-1802^{*}$ & & $-0.555^{*}$ & \\
\hline JARDIM & 8 & 35519 & 28223 & 10.636 & 8.644 \\
\hline BOX & - & 87545 & $70992^{*}$ & 26.908 & $22.451^{*}$ \\
\hline DEPEMP & - & 124132 & 135354 & 37.149 & 40.901 \\
\hline MOBILIA & - & 82613 & 60246 & 25.769 & 19.480 \\
\hline TIPO & - & 43861 & & 11.182 & \\
\hline CONSTANTE & & $-42963^{* *}$ & $24782^{* *}$ & $-33.957^{*}$ & 25.570 \\
\hline R $^{2}$ & & 0.711 & 0.762 & 0.713 & 0.758 \\
\hline F & & 74.869 & 97.253 & 86.948 & 109.377 \\
\hline $\mathrm{n}$ & & 252 & 252 & 252 & 252 \\
\hline $\mathrm{k}$ & & 8 & 8 & 7 & 7 \\
\hline "OUTLIERS" & & 6 & 6 & 6 & 8 \\
\hline
\end{tabular}

(nivel de significância: **:>10\%; *:5\%, outros: $1 \%$ )

Tabela 4- Regressões em CrS e USS, para imóveis situados até 2,24 KM do centro da cidade 


\begin{tabular}{|c|c|c|c|c|c|}
\hline VARIAVEL & FATOR & $\operatorname{cr} \$(1)$ & $\operatorname{crs}(2)$ & USs $(1)$ & USS $(2)$ \\
\hline AREA & 1 & & 5113 & & 1.587 \\
\hline QUARTOS & 1 & 123650 & & 36.751 & \\
\hline BANHEIROS & 2 & 122089 & $117+28$ & 35.394 & 32.394 \\
\hline ANDARES & 3 & 19607 & 139013 & +670 & 3.571 \\
\hline DIA & 6 & 3454 & 3401 & & \\
\hline ESTADO & 7 & & & $0.36,2$ & \\
\hline PREDIOS & 8 & -35974 & & & \\
\hline DENSIDADE & 9 & $529 *$ & & & \\
\hline DCENTRO & 9 & & & $-3.996 *$ & \\
\hline DSUPER & I) & & 13580 & & 4.482 \\
\hline $\mathrm{BOX}$ & $\therefore$ & 152306 & 6,3427 & 4.050 & 17.868 \\
\hline BAIRKO & - & $1933 *$ & $10+1 \cdots$ & $0.398^{\circ}$ & \\
\hline COBERTURA & - & $1551+68$ & 904129 & 42.3 .323 & $238 \times 661$ \\
\hline FAVD & $\therefore$ & .144260 & $-1123 \times 1$ & .42 .102 & -36.370 \\
\hline MORII.IA & . & 256505 & 174775 & 72.157 & 48.023 \\
\hline TIPO & - & $131+81$ & 72406 & 36,359 & 22.044 \\
\hline CONSTANTE & & -.577046 & -405783 & -122412 & $-112+53$ \\
\hline $\mathrm{R}^{2}$ & & 0.847 & 0.874 & 0.845 & 0.875 \\
\hline$F$ & & $1111.99 x$ & $151.92 \mathrm{~K}$ & 119.310 & 169.532 \\
\hline (n) & & 252 & 252 & 252 & 252 \\
\hline $\mathbf{k}$ & & 12 & III & 11 & 10 \\
\hline "OISILIERS" & & 11 & 12 & 11 & $\overrightarrow{12}$ \\
\hline
\end{tabular}

(nivel de significância: **:>10\%; *.5\%; outros:1\%)

\section{Tabela 5 - Regressões em CRS e USS, para Imóveis Situados a Mais de $2.24 \mathrm{Km}$ do} Centro da Cidade

\section{Conclusões}

Foram compiladas equações que atingiram mais de $80 \%$ de cxplicação da variabilidade dos valores de alugucl. Estes resultados, aliados ao controle de resíduos c de colinearidade, confirmam a validade destes modelos. Pode-se concluir que a análisc cicntifica realizada permitc confiança para a generalização dos resultados.

Foram detectadas diversas variáveis importantes para a formação do valor, como distância aos "shopping centers" $\mathrm{c}$ cxistência de habitações favcladas nas proximidades. Os modelos com valores $\mathrm{cm}$ cruzciros ou convertidos cm dólares foram gerilmente semelhantes.
Ressalta-se o bom ajustamento dos modelos para o grupo geral, que podem ser usados para decrminar o aluguel de qualquer imóvel no periodo csludado, represcntando a mćdia dos alugućis da cidade coln uma cquação apenas. Ncstc sentido, podem ser considerados modelos inticos.

Por outro hado. os modelos apresentam baslante distinção conforme o conjunto de imóveis considerado. As cquações para o conjunto geral sĩo diferentes daquelas compiladas para parcelas deste conjunto. Isto ocorre cm parte por cauusa de distinções causadas pela consideração de sub-mercados, mas, mesmo assim, esperaval-se a existência de conjuntos de variaivcis scmclhantes, o que não ocorreı. 
Portanto, a hipótese da existência de um modelo único não pode ser confirmada totalmente, embora haja indicações favoráveis.

\section{ANEXO}

\section{Descrição das Variáveis}

\begin{tabular}{|c|c|c|c|c|c|c|}
\hline VARIÁVEL & DESCRICAO & MIN & MAX & MÉDIA & UNID & FONTE \\
\hline CRUZEIRO & valer de aluguel of miado, em Crs & 120 & 3500 & 411 & Crs & Jornal/mobiliária \\
\hline DOI.AR & valor do alu guel cenverido em tiss & 33 & 998 & 121 & IISS & cotaciòes diàinas \\
\hline APTOS & Intil de apartamentos mo prédio & 1 & .308 & 49.3 & un & Vistorias \\
\hline AREA & drea total do imóvel & 21 & 347 & 65.7 & $\mathbf{m}^{2}$ & PMPA \\
\hline BAIRRO & qualificaça base ada na renda medis dis populacia & 0 & 100 & 27.8 & $\cdot$ & IJGE, 1980 \\
\hline BANHEIKOS & nimero de banheiros & 1 & $\mathbf{3}$ & 1.04 & 411 & Jomil//mobiliarias \\
\hline $130 \mathrm{x}$ & indica existiticia de hox ou gar agem & 0 & 2 & 0.13 & Un & Jomal/numbiliarias \\
\hline COBERTURA & indica exjstencis de cobertura & 0 & 1 & - & $\cdot$ & Jornalilnobiliárias \\
\hline DCENTRO & distincia ao senuro (pontn de effertncis; PMPA] & 0.16 & 1.3 .20 & .111 & $\mathrm{~km}$ & 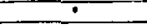 \\
\hline DF.PEMP & indica existéncia de dependèncias de empregada & 0 & 1 & $\cdot$ & - & Joms//mobiliarias \\
\hline DIA & dia da oferta $(31 / 5 / 52=1 ; 2 n / 92-33)$ & 1 & $\mathbf{3 1}$ & 20.4 & - & Jomal/lmubiliznias \\
\hline DSIIOP & distàxia an "shopring cemer" mais róximo & 0.33 & 8.68 & 2.42 & I.III & -7 \\
\hline DSUPER & 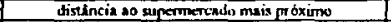 & 0.05 & 4.63 & 4.22 & $\mathrm{~km}$ & + \\
\hline ESQUINA & inkiveis siluados $\mathrm{cm}$ esiuuina & 0 & 1 & + & - & Vistorizs \\
\hline ESTAINO & estado de conservacto do predio & 0 & 100 & 31 & $\therefore$ & Vistorias \\
\hline FAVD & indica exirtiencia de favelas no entomo & 0 & 1 & $\therefore$ & - & Vistorias \\
\hline JARDIM & lipo de jardim do prédio (nentum =0; prende $=4$ ) & $\mathbf{0}$ & 4 & 0.97 & $\dot{-}$ & Vistotias \\
\hline MOBILIA & indies incluslo de mobifie no contralo & 0 & 2 & 0.05 & 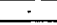 & Junual/mobiliarias \\
\hline NAM & número de arartanierias pue andur & 1. & 23.64 & 6.35 & tull & APTOSIANDARES \\
\hline PREDIOS & numero de pródins ignisis & 1 & 30 & 2.38 & un & Vistorias \\
\hline QUALIDADE & padrlo de construçio do predio & $\mathbf{0}$ & 100 & 44.3 & $\therefore$ & Vistotias \\
\hline QUARTOS & nurmero de domutónios & 0 & 4 & 1.38 & un & Jomnl'Imobiliarias \\
\hline TIPO & gualifica za para a construça atritu ida mo "flabilese" & 2 & 3 & 3.15 & $\cdot$ & PAIPA \\
\hline ESOS & Dercentual ike wan sesickencial no entomo supeniox a $50 \%$ & o & 1 & . & $=$ & Vistorias \\
\hline
\end{tabular}

(*Variávcis terminadas pelo autor) 


\section{Referências Bibliográficas}

ASSOCIAÇÃO BRASILEIRA DE NORMAS TÉCNICAS. Avaliaçôes de imóveis urbanos: NB-502. Rio de Janeiro, 1989.

BALCHIN, P. N. Housing policy and housing necds. London: McMillan, 1981.

: KIEVE, J.L. Urban land cconomics. 3ed. London: McMillan, 1986.

COChram, W. G. Técnicas de amostragem. Rio de Janeiro: Fundo de Cultura, 1965.

FRANCHI, C. C. Avaliação das caracteristicas que contribucm para a formação do valor de apartamentos na cidade de Porto Alegre. Porto Alegre: Curso de Pós-Graduação em Engcnharia Civil/UFRGS. 1991. Disscrtação (Mcstrado cm Engenharia).

FUNDAÇÃOINSTITUTOBRASILEIRO DE GEOGRAFIA E ESTATISTICA. Ceısos brasileiros - tabulações espociais: 1980 c 1991. Rio de Janeiro: FIBGE.

GONZÁLEZ, M. A. S. A formação do valor dos aluguéis de apartamentos residenciais na cidade de Porto Alegre. Porlo Alegre: Curso de Pós-Graduação cm Engenharia Civil/UFRGS, 1993. Disscrtação (Mestrado cm Engenharia).

GOODALL, B. The cconomics of urban areas. Oxford: Pergamon, 1972.

HARMANN, H. H. Modern factor analysis. 3ed. Chicago, University of Chicago, 1976.

JOHNSTON, J. Métodos econométricos. São Paulo: Aulas, 1977.

KAIN, J. F.; QUIGLEY, J. M. Measuring the Value of Housing Quality. Journal of the American Statistical Association, Washington: American Statistical Association, v.65, p.532-548, Junc 1970.

KMENTA, J. Elcmentos de econometria. São Paulo: Atlas, 1978.

KONIJN, H. S. Statistical theory of sample survey design and analysis. Amsicrdam: North-Holland, 1973.

LAVENDER. S D. Economics for builders and surveyors. Essex,UK: Longman, 1990.

LEAN, W.; GOODALL, B. Aspects of land economics. London: The Estates Gazette, 1966.

LUCENA, J. M. P. O mercado habitacional no Brasil. Rio de Janeiro: FGV, 1985.

MADDALA, G.S. Econometrics. New York: McGraw-Hill, 1977.

MORTON, T. G. Factor analysis, multicollinearity, and regression appraisal models. The Appraisal Joumal. Chicago: 
American Institute of Real Estate Appraisers, v.45, p.578-587, Oct. 1977.

MUTH, R. F. Urban cconomic problems. New York: Harper \& Row, 1975.

NETER; J; WASSERMAN, W. Applied lincar statistical models. Homewood, IL: Richard D. Invin, 1974.

NIE, N. H. ct al. SPSS - Statistical package for the social sciences. 2ed. New York: McGraw-Hill, 1975.

RAJ, D. The design of sample surveys. New York: McGraw-Hill, 1972.

ROBINSON, R. Housing economics and public policy. London: MacMillan, 1979 .

ROSEN, S. Hedonic prices and implicit markets: Product differentiation in pure competition. Journal of Political Economy,
Chicago: University of Chicago, n.82, p.34-55, Jan. 1974.

SUDMAN, S. Applicd sampling. New York: Academic Press, 1976.

WEIMER, A. M.; HOYT, $\mathrm{H}$. Principles of urban real estate. ed.rev. New York: Ronald Press, 1948.

WEISBERG, S. Applied lincar regression. 2ed. New York: John Wiley, 1985.

WILKINSON, R.K. and ARCHER, C. A. Measuring the determinanints of relative house prices. Environment and Planning. v.5, n.3, p.357-367, May 1973.

WITTE, A. D.; SUMKA, H. J.: EREKSON, H. All estimatc of a structural hedonic price model of the housing market: An application of Rosen's theory of implicit markcts. Econometrica, v.47, 11.5, p.1151-1173, Sept.1974. 